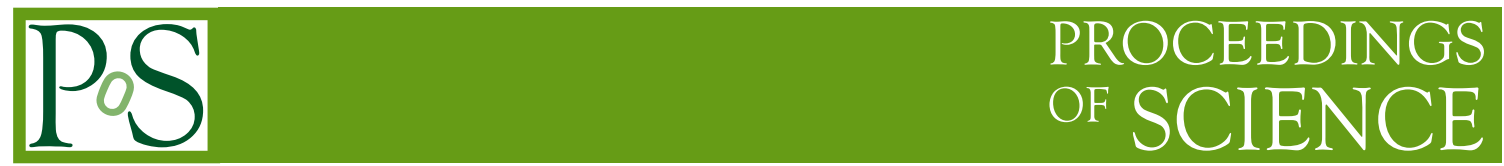

\title{
Center Vortices and Topological Charge
}

\author{
Roman Höllwieser*, Thomas Schweigler, Manfried Faber \\ Atomic Institute/Nuclear Physics, Vienna University of Technology, Wiedner Hauptstr. 8-10, \\ A-1040 Vienna, Austria
}

\section{Urs M. Heller}

American Physical Society, One Research Road, Ridge, NY 11961, USA

\begin{abstract}
Center vortices explain color confinement and are also of paramount importance for spontaneous chiral symmetry breaking. Center vortices contribute to the topological charge density through intersection and writhing points, but also through their color structure. The prototype of the later contribution is the spherical vortex introduced in previous articles of our group. Here, we analyze the different topological charge contributions from classical $S U(2)$ center vortices with shapes of planes and spheres in more detail. Our results show that the interpretation of topological charge in the background of center vortices is rather subtle.
\end{abstract}

Xth Quark Confinement and the Hadron Spectrum,

October 8-12, 2012

TUM Campus Garching, Munich, Germany

*Speaker. 


\section{Introduction}

The center vortex model $[1,2,3,4,5,6]$ seems to be a very promising candidate to explain the phenomena that dominate the infrared regime. In lattice QCD it was shown $[7,8,9]$ that vortices (quantized magnetic fluxes) condense in the vacuum and compress the electric flux between quark and antiquark to a string leading to confinement. Numerical simulations have indicated that vortices could also account for phenomena related to chiral symmetry, such as causing topological charge fluctuations and spontaneous chiral symmetry breaking (SCSB) [10, 11, 12, 13]. In particular, [14] states that the topological charge of a gauge field can be determined from the shape of P-vortices, i.e. from vortex intersections and writhing points. In previous articles of our working group $[15,16$, 17], we introduced another source of topological charge, the spherical SU(2) center vortex, which contributes through its color structure. However, this color structure contribution is nontrivial and its detection requires some effort.

The topological charge $Q_{U}$ of a lattice gauge field $U$ is determined by the integral

$$
Q_{U}=-\frac{1}{16 \pi^{2}} \int d^{4} x \operatorname{Tr}\left[\tilde{F}_{\mu v} F_{\mu v}\right]
$$

where $F_{\mu \nu}$ is expressed in terms of the plaquette field

$$
P_{\mu v}=U_{\mu}(x) U_{v}(x+\mu) U_{\mu}^{\dagger}(x+v) U_{v}^{\dagger}(x) .
$$

This expression is derived in the continuum from the transition between vacua with different winding numbers [18, 19] $Q=\int_{S_{3}} J_{\mu} d \sigma_{\mu}$, with $J_{\mu}=-\frac{1}{8 \pi^{2}} \varepsilon_{\mu v \alpha \beta} \operatorname{Tr}\left(A_{v} \partial_{\alpha} A_{\beta}+2 / 3 A_{v} A_{\alpha} A_{\beta}\right)$. Since $F_{\mu \nu} \tilde{F}_{\mu \nu}=\partial_{\mu} J_{\mu}, Q$ can be re-expressed as the above volume integral (1.1). On the lattice, continuity in space is lost and it seems that one should be able to view any lattice field configuration as being a discrete copy of a smooth continuum configuration. This would always be topologically trivial, since $F \tilde{F}$ is a total derivative. Nonzero $Q_{U}$ may come from field configurations containing gauge singularities $[20,21,22]$. Another possibility to analyze the topology of a gauge field is given by the Atiyah-Singer index theorem [23, 24, 25, 26]. It states that in the continuum the topological charge of a gauge field configuration equals the index of the Dirac operator in this gauge field background. For the overlap Dirac operator [26, 27] in the fundamental representation the index is given by ind $D[A]=n_{-}-n_{+}=Q_{D}$, where $n_{-}$and $n_{+}$are the number of leftand right-handed zeromodes. The lattice version of the index theorem is only valid as long as the gauge field is smooth enough and satisfies a so-called "admissibility" condition. It requires that the plaquette values $U_{\mu v}$ are bounded close to $\mathbb{1}$, the value for very smooth gauge fields. Sufficient, but not necessary bounds for the "admissibility" of the gauge field are $\left\|1-U_{\mu v}\right\|<1 / 30$ [20], or $\left\|1-U_{\mu v}\right\|<[6(2+\sqrt{(2)})]=0.04882[28]$.

The spherical center vortex fulfills the admissibility condition but clearly shows a discrepancy between the integral of $F \tilde{F}$ and the topological charge derived from the lattice index theorem. This problem was identified to be a discretization error of the $F \tilde{F}$ definition for this special case [17], and could be resolved by using topologically motivated topological charge definitions. Recently we found a way to regularize the spherical vortex [29]. Here we want to analyze these issues in more detail and start with the discussion of plane vortices in different $U(1)$-subgroups of $S U(2)$. 


\section{Colorful Vortex Intersections}

In $S U$ (2) lattice gauge theory, plane vortices parallel to two of the coordinate axes are defined by links varying in a $U(1)$ subgroup of $S U(2)$. This $U(1)$ subgroup is generated by one of the Pauli matrices $\sigma_{i}$, i.e. $U_{\mu}=\exp \left(\mathrm{i} \phi \sigma_{i}\right)$. The direction of the flux and the orientation of the vortices are determined by the gradient of the angle $\phi$, which we choose as a piecewise linear function of the coordinate perpendicular to the vortex. The explicit functions for $\phi$ are given in Eq. (2.1) of [30]. Upon traversing a vortex sheet, the angle $\phi$ increases or decreases by $\pi$ within a finite thickness of the vortex. Center projection leads to a (thin) P-vortex at half the thickness [7]. If we consider these thick, planar vortices intersecting orthogonally, each intersection carries a topological charge with modulus $|Q|=1 / 2$, whose sign depends on the relative orientation of the vortex fluxes [31]. The plaquette definition simply discretizes the continuum (Minkowski) expression of the Pontryagin index to a lattice (Euclidean) version of the topological charge definition

$$
Q_{U}=-\frac{1}{16 \pi^{2}} \int d^{4} x \operatorname{tr}\left[\tilde{F}_{\mu v} F_{\mu v}\right]=-\frac{1}{32 \pi^{2}} \int d^{4} x \varepsilon_{\mu v \alpha \beta} \operatorname{tr}\left[F_{\alpha \beta} F_{\mu v}\right]=\frac{1}{4 \pi^{2}} \int d^{4} x \vec{E} \cdot \vec{B} .
$$

If the contributing vortex plaquettes lie in the same $U(1)$ subgroup of $S U(2)$, then parallel vortex crossings give $Q_{U}=1 / 2$ and antiparallel vortex crossings give $Q_{U}=-1 / 2$. Using different $U(1)$ subgroups for the individual vortex sheets however, $Q_{U}$ for such intersections gives zero. The action of orthogonal color vector intersections on the other hand is very high since we get maximally nontrivial plaquettes

$$
P \approx\left(-\mathrm{i} \sigma_{k}\right)\left(-\mathrm{i} \sigma_{l}\right) \mathrm{i} \sigma_{k} \mathrm{i} \sigma_{l}=\left(\sigma_{k} \sigma_{l}\right)\left(\sigma_{k} \sigma_{l}\right)=\mathrm{i} \sigma_{m} \mathrm{i} \sigma_{m}=-\mathbb{1}
$$

Therefore such configurations are suppressed in the QCD vacuum, but smooth color rotations may definitely contribute to the topological charge.

\section{The Colorful Spherical Vortex}

The spherical vortex of radius $R$ and thickness $\Delta$ was introduced in [15] and analyzed in more detail in [32]. It is constructed with the following links:

$$
U_{\mu}\left(x^{v}\right)=\left\{\begin{array}{ll}
\exp \left(\mathrm{i} \alpha\left(\left|\vec{r}-\vec{r}_{0}\right|\right) \vec{n} \cdot \vec{\sigma}\right) & t=t_{i}, \mu=4 \\
\mathbb{1} & \text { elsewhere }
\end{array} \text { with } \quad \vec{n}(\vec{r}, t)=\frac{\vec{r}-\vec{r}_{0}}{\left|\vec{r}-\vec{r}_{0}\right|}\right.
$$

where $\vec{r}$ is the spatial part of $x_{v}$ and the profile function $\alpha$ is either one of $\alpha_{+}, \alpha_{-}$, which are defined by

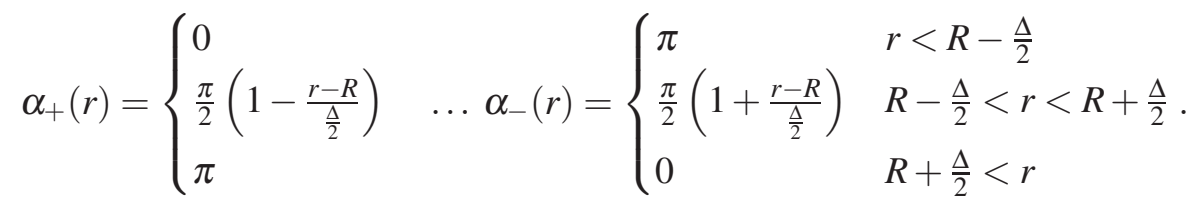

This means that all links are equal to $\mathbb{1}$ except for the $t$-links in a single time slice at fixed $t=t_{i}$. The phase changes from 0 to $\pi$ from inside to outside for $\alpha_{+}(r)$ (or vice versa for $\alpha_{-}(r)$ ). The 
graph of $\alpha_{-}(r)$ is plotted in Fig. 2 in [15], giving a hedgehog-like configuration, since the color vector $\vec{n}$ points in the "radial" direction $\vec{r} / r$ at the vortex radius $R$. The hedgehog-like structure is crucial for our analysis, the $t$-links of the spherical vortices fix the holonomy of the time-like loops, defining a map $U_{t}\left(\vec{x}, t=t_{i}\right)$ from the $x y z$-hyperplane at $t=t_{i}$ to $S U(2)$. Because of the periodic boundary conditions, the time slice has the topology of a 3-torus. But, actually, we can identify all points in the exterior of the 3 dimensional sphere since the links there are trivial. Thus the topology of the time slice is $\mathbb{R}^{3} \cup\{\infty\}$ which is homeomorphic to $S^{3}$. A map $S^{3} \rightarrow S U(2)$ is characterized by a winding number

$$
N=-\frac{1}{24 \pi^{2}} \int d^{3} x \varepsilon_{i j k} \operatorname{Tr}\left[\left(U^{\dagger} \partial_{i} U\right)\left(U^{\dagger} \partial_{j} U\right)\left(U^{\dagger} \partial_{k} U\right)\right],
$$

resulting in $N=-1$ for positive and $N=+1$ for negative spherical vortices. With this assignment the index of the Dirac operator and the topological charge after cooling coincide with this winding number $N$. Obviously such windings, given by the holonomy of the time-like loops of the spherical vortex, influence the index theorem [33,34], which gives the correct definition of topological charge. We would like to underline that for the spherical vortex with the hedgehog structure there is no discontinuity in the link variables, not in the center of the three-dimensional spherical vortex nor at the lattice boundary. In both regions the link variables are center elements as necessary for a center vortex. In spite of the hedgehog structure at the two-dimensional center projected vortex sphere there is no singularity due to the full covering of $S^{3}$ by the link variables. In other words one can explain the configuration with $t$-links of one time slice rotating from the "south pole" $(-\mathbb{1})$ to the "north pole" $(+\mathbb{1})$ of $S^{3}$ in radial direction from the center to the boundary, via color vectors $\vec{n} \vec{\sigma}$ given by the spatial components $\vec{n}=\vec{r} / r$ of the radius vector. Hence, at the center of the vortex sphere the links belong to the "south pole" of $S^{3}$, at the two-dimensional vortex surface to the "equator" and at the boundary of the time slice to the "north pole" of $S^{3}$. In other words, the links $U_{t}(x, y, z)$ define a smooth nontrivial mapping of $S_{3} \cong S U(2)$ to $R_{3} \cup \infty \cong S_{3}$ which does not contain any singularity. The index of the overlap Dirac operator is nonzero, resulting in $Q_{D}=\mp 1$, for $\alpha_{ \pm}$. On a $136^{3} \times N_{t}$ lattice the plaquettes for the spherical vortex satisfy the "admissibility" condition. In fact, the plaquettes get smaller and smaller the bigger the lattice, if we choose $R$ and $\Delta$ to be proportional to the lattice size. We can even get rid of negative links with proper gauges (Landau gauge), ending up in a lattice gauge field with no sign of hiding a singularity at all. However, since only links in the time direction are different from $\mathbb{1}$ for this spherical vortex configuration, the topological charge $Q_{U}$ determined from any lattice version of $F \tilde{F}$ vanishes for this spherical vortex configuration.

Next, we apply standard cooling to the spherical vortex configuration, in order to understand the discrepancy. For many cooling steps, the index of the Dirac operator does not change, but the topological charge quickly rises close to $Q_{U}=\mp 1$ for $\alpha_{ \pm}$while the action $S$ reaches a (nonzero) plateau. So, the index of the overlap Dirac operator agrees with the topological charge via $F \tilde{F}$ $\left(Q_{U}=Q_{D}\right)$ after some cooling. In Fig. 1a) we plot the cooling history for a spherical vortex on a $40^{4}$ lattice. For comparison we also plot the topological charge of an instanton during cooling, which looks pretty much the same as for the spherical vortex. In fact, the action and topological charge densities spread over more and more time slices, developing a hyperspherical distribution like standard instantons. We conclude that our spherical vortex develops an instanton-like structure during cooling, in agreement with [35], stating that the Hausdorff dimension of regions where 

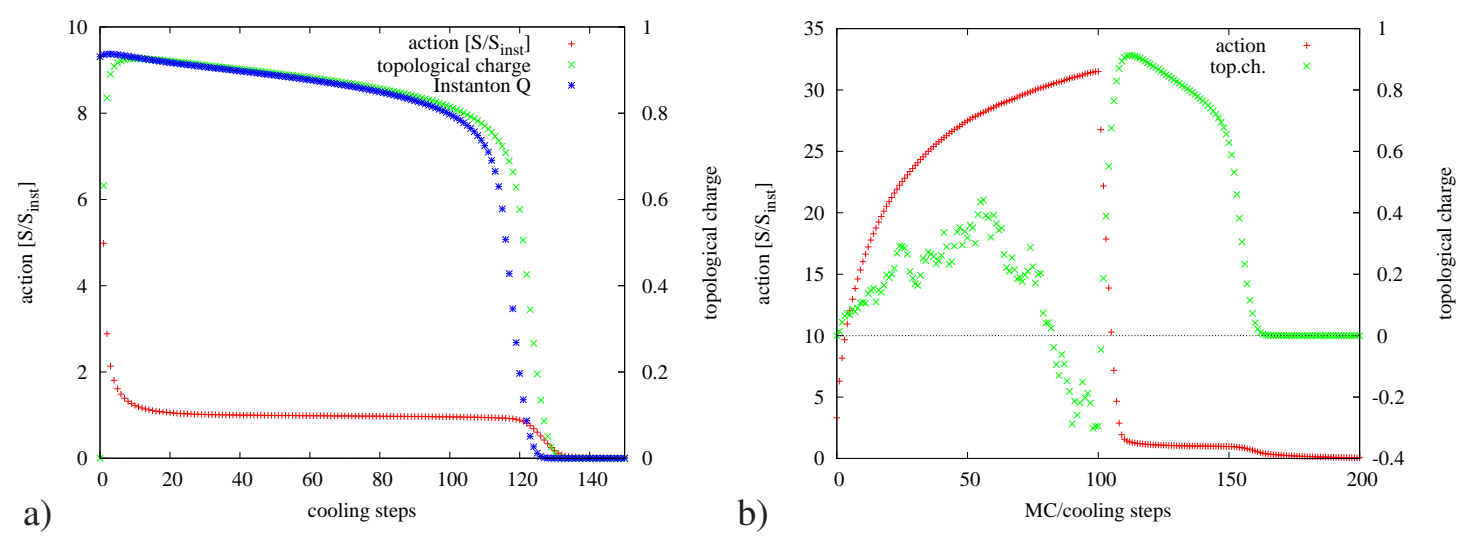

Figure 1: a) Cooling of a spherical vortex on a $40^{4}$ lattice. The topological charge rises from zero to close to one for $\alpha=\alpha_{-}$(right scale) while the action $S$ (in units of the one-instanton action $S_{\text {inst }}$ ) reaches a plateau (left scale). For comparison we also plot the topological charge during cooling of an instanton. b) Monte Carlo (Metropolis) update and cooling of a spherical vortex on a $16^{4}$ lattice. After $100 \mathrm{MC}$ steps, only after some additional cooling $F \tilde{F}$ reveals the same topological charge $Q_{U}=Q_{D}$ as the Dirac operator.

the topological charge is localized, tends towards the total space dimensions. However, the vortex structure of our initial configuration is removed after a few cooling steps, i.e., the spherical vortex shrinks very quickly. This is in agreement with the fact that the vortex content of a single instanton is shrunk to a point at the center of the instanton [36, 37], but it clearly shows that cooling significantly changes the content of the initial gauge configuration. Further we apply some Monte Carlo steps to the spherical vortex configuration using the Metropolis algorithm with a small spread, i.e. adding small quantum fluctuations, and analyze the vortex structure and topological charge. Fig. 1b) shows the action and topological charge during 100 Metropolis and another 100 cooling steps. The action rises during the Monte Carlo update and the spherical vortex percolates over the whole lattice while the topological charge fluctuates around zero. The index of the lattice Dirac operator however indicates topological charge $Q_{D}=\mp 1$ for $\alpha_{ \pm}$and some cooling after the Monte Carlo update also reveals the topological winding in $Q_{U}$.

\section{Regularization of the Spherical Vortex}

The problem with the gluonic topological charge definition $Q_{U}$ of the original spherical vortex is clearly related to the singular gauge transformation, i.e., only time-like links of a single time slice are nontrivial, which can also be regarded as some kind of discretization effect. In order to get rid of this, one has to apply a gauge transformation $\Omega\left(x^{v}\right)$, with spatial dependence $g(\vec{r})$ is given by the original, nontrivial link configuration of the original spherical vortex, Eq. (3.1), i.e.,

$$
g(\vec{r})=\exp \left(\mathrm{i} \alpha\left(\left|\vec{r}-\vec{r}_{0}\right|\right) \vec{n} \cdot \vec{\sigma}\right) \quad \text { with } \quad \vec{n}(\vec{r}, t)=\frac{\vec{r}-\vec{r}_{0}}{\left|\vec{r}-\vec{r}_{0}\right|},
$$

where $\vec{r}$ is again the spatial part of $x_{v}$ and the profile function $\alpha$ was defined in Eq. (3.2). With this lattice gauge transformation

$$
\Omega\left(x^{v}\right)= \begin{cases}g(\vec{r}) & \text { for } t_{i}<t \leq t_{f} \\ \mathbb{1} & \text { else }\end{cases}
$$


the spherical vortex becomes

$$
U_{\mu}\left(x^{v}\right)=\left\{\begin{array}{ll}
g(\vec{r}+\hat{\mu}) g(\vec{r})^{\dagger} & \text { for } \mu=1,2,3 ; t_{i}<t \leq t_{f} \\
g(\vec{r})^{\dagger} & \text { for } \mu=4 ; t=t_{f} \\
\mathbb{1} & \text { else }
\end{array} .\right.
$$

This means we gauge transform the original, nontrivial time-like links at the initial time slice $t=t_{i}$ away to some final time slice $t=t_{f}$ and are left with some pure gauge field between time slices $t_{i}<t \leq t_{f}$. From this it becomes clear that the spherical vortex represents a transition (in the temporal direction) between two pure gauge fields. The transition occurs between $t=t_{i}$ and $t=t_{i}+1$. No transition occurs between $t=t_{g}$ and $t=t_{g}+1$ since all plaquettes there are trivial. For $t \leq t_{i}$ the pure gauge field is trivial, i.e., the winding number $N=0$. For $t>t_{i}$ the pure gauge field is generated by the hedgehog gauge transformation, Eq. (4.1), which has winding $N=1$ [38]. This means that in the continuum limit, the spherical vortex (representing a transition between a vacuum with winding number 0 and 1) has topological charge $Q=1$ [38]. It can be regarded as a squeezed instanton with center vortex structure. However, since we only did a gauge transformation of the original field, $Q_{U}$ still yields zero topological charge. The actual vacuum to vacuum transition (carrying the entire topological charge) occurs in a single time slice $\left(t=t_{i}\right)$ and therefore cannot be resolved by the lattice. What we have to do is to smooth out the transition over more than one time slice, by rising the nontrivial links in Eq. (4.3) to the power of some function $f(t)$, which is monotonically rising from 0 to 1 between time slices $t_{i}$ and $t_{f}$. Spreading our regularized, spherical vortex over a few time slices, the topological charge $Q_{U}$ gets close to 1 , as expected. For more details, see $[29,39]$.

\section{Conclusion}

We presented some issues related to problems defining topological charge in the background of classical center vortices on the lattice. First, planar vortex sheets are constructed by $U(1)$ rotations in a way that they intersect orthogonally. These intersections are known to carry a topological charge $Q= \pm 1 / 2$, but if the gauge rotations are defined in different $U(1)$-subgroups of $S U(2)$, $Q_{U}=F \tilde{F}$ fails to detect the topological charge contributions. The use of the Dirac operator is not safe in this case because of maximally nontrivial plaquettes, which seem to suppress such configurations in the functional integral. Further, the colorful, spherical center vortices introduced in previous works is analyzed in more detail. The discrepancy between $Q_{U}$ and the index of the overlap Dirac operator $\left(Q_{D}\right)$ equals the winding number of the vortex spheres when they are regarded as maps $S^{3} \rightarrow S U(2)$. The problem arises due to the fact that center vortices are based on singular gauge transformations, which leads to a discretization error of the gluonic topological charge definition. We show how to regularize the spherical vortex, by spreading the vacuum to vacuum transition of the singular gauge transformation over several time slices, which resolves the discrepancy between $Q_{U}$ and $Q_{D}$. This procedure is similar to what happens during cooling the spherical vortex, it transforms into some instanton like structure. We conclude that the interpretation of topological charge via $F \tilde{F}$ is rather subtle in the background of center vortices. 


\section{Acknowledgments}

We want to thank Martin Lüscher, Štefan Olejník and Mithat Ünsal for helpful discussions. This work was partially supported by the Austrian Science Fund ("Fonds zur Förderung der Wissenschaften”, FWF) under contract P22270-N16 (R.H.).

\section{References}

[1] G. 't Hooft. On the phase transition towards permanent quark confinement. Nucl. Phys., B138:1, 1978.

[2] P. Vinciarelli. Fluxon solutions in nonabelian gauge models. Phys. Lett., B78:485-488, 1978.

[3] Tamiaki Yoneya. Z(n) topological excitations in yang-mills theories: Duality and confinement. Nucl. Phys., B144:195, 1978.

[4] J. M. Cornwall. Quark confinement and Vortices in massive gauge invariant QCD. Nucl. Phys., B157:392, 1979.

[5] G. Mack and V. B. Petkova. Comparison of Lattice Gauge Theories with gauge groups Z(2) and SU(2). Ann. Phys., 123:442, 1979.

[6] H. B. Nielsen and P. Olesen. A Quantum Liquid Model for the QCD Vacuum: Gauge and Rotational Invariance of Domained and Quantized Homogeneous Color Fields. Nucl. Phys., B160:380, 1979.

[7] L. Del Debbio, and M. Faber, and J. Greensite, and Š. Olejník. Center dominance and Z(2) vortices in SU(2) lattice gauge theory. Phys. Rev., D55:2298-2306, 1997.

[8] L. Del Debbio, and M. Faber, and J. Greensite, and Š. Olejník. Center dominance, center vortices, and confinement. [arXiv:hep-lat/9708023].

[9] Tamas G. Kovacs and E. T. Tomboulis. Vortices and confinement at weak coupling. Phys. Rev., D57:4054-4062, 1998.

[10] Philippe de Forcrand and Massimo D'Elia. On the relevance of center vortices to QCD. Phys.Rev.Lett., 82:4582-4585, 1999.

[11] C. Alexandrou, P. de Forcrand, and M. D'Elia. The role of center vortices in QCD. Nucl. Phys., A663:1031-1034, 2000.

[12] Michael Engelhardt. Center vortex model for the infrared sector of Yang-Mills theory: Quenched Dirac spectrum and chiral condensate. Nucl.Phys., B638:81-110, 2002.

[13] R. Höllwieser, and M. Faber, and J. Greensite, and U.M. Heller, and Š. Olejník. Center Vortices and the Dirac Spectrum. Phys. Rev., D78:054508, 2008.

[14] H. Reinhardt and M. Engelhardt. Center vortices in continuum yang-mills theory. In Wolfgang Lucha and Khin Maung Maung, editors, Quark Confinement and the Hadron Spectrum IV, pages 150-162. World Scientific, 2002.

[15] G. Jordan, and R. Höllwieser, and M. Faber, and U.M. Heller. Tests of the lattice index theorem. Phys. Rev., D77:014515, 2008.

[16] R. Höllwieser, and M. Faber, and U.M. Heller. Lattice Index Theorem and Fractional Topological Charge. [arXiv:hep-lat/1005.1015]. 
[17] R. Höllwieser, and M. Faber, and U.M. Heller. Critical analysis of topological charge determination in the background of center vortices in SU(2) lattice gauge theory. Phys.Rev., D86:014513, 2012.

[18] A. A. Belavin, Alexander M. Polyakov, A. S. Schwartz, and Yu. S. Tyupkin. Pseudoparticle solutions of the Yang-Mills equations. Phys. Lett., B59:85-87, 1975.

[19] Michael E. Peskin. Chirality Conservation in the Lattice Gauge Theory. 1978. Ph.D. Thesis.

[20] M. Lüscher. Topology of Lattice Gauge Fields. Commun.Math.Phys., 85:39, 1982.

[21] Peter Woit. Topoligical Charge in Lattice Gauge Theory. Phys.Rev.Lett., 51:638, 1983.

[22] I.A. Fox, J.P. Gilchrist, M.L. Laursen, and G. Schierholz. The Topological Charge in SU(2) Lattice Gauge Theory. Phys.Rev.Lett., 54:749, 1985.

[23] M. F. Atiyah and I. M. Singer. The Index of elliptic operators. 5. Annals Math., 93:139-149, 1971.

[24] A. S. Schwarz. On Regular Solutions of Euclidean Yang-Mills Equations. Phys. Lett., B67:172-174, 1977.

[25] Lowell S. Brown, Robert D. Carlitz, and Choon-kyu Lee. Massless Excitations in Instanton Fields. Phys. Rev., D16:417-422, 1977.

[26] Rajamani Narayanan and Herbert Neuberger. A construction of lattice chiral gauge theories. Nucl. Phys., B443:305-385, 1995.

[27] Herbert Neuberger. Exactly massless quarks on the lattice. Phys. Lett., B417:141-144, 1998.

[28] Herbert Neuberger. Bounds on the Wilson Dirac operator. Phys. Rev., D61:085015, 2000.

[29] T. Schweigler, R. Höllwieser, M. Faber, and U.M. Heller. Colorful SU(2) center vortices in the continuum and on the lattice. [arXiv:hep-lat/1212.3737].

[30] R. Höllwieser, and M. Faber, and U.M. Heller. Intersections of thick Center Vortices, Dirac Eigenmodes and Fractional Topological Charge in SU(2) Lattice Gauge Theory. JHEP, 1106:052, 2011.

[31] M. Engelhardt and H. Reinhardt. Center projection vortices in continuum Yang-Mills theory. Nucl. Phys., B567:249, 2000.

[32] R. Höllwieser, and M. Faber, and U.M. Heller. Lattice Index Theorem and Fractional Topological Charge. 2010.

[33] Tom M. W. Nye and Michael A. Singer. An $L^{2}$-Index Theorem for Dirac Operators on $S^{1} * R^{3} .2000$, [arXiv:math/0009144].

[34] Erich Poppitz and Mithat Unsal. Index theorem for topological excitations on $R^{3} * S^{1}$ and Chern-Simons theory. JHEP., 0903:027, 2009.

[35] P.V. Buividovich, T. Kalaydzhyan, and M.I. Polikarpov. Fractal dimension of the topological charge density distribution in SU(2) lattice gluodynamics. Phys.Rev., D86:074511, 2012.

[36] H. Reinhardt and T. Tok. Merons and instantons in laplacian abelian and center gauges in continuum Yang-Mills theory. Phys. Lett., B505:131-140, 2001.

[37] Falk Bruckmann and Michael Engelhardt. Writhe of center vortices and topological charge: An Explicit example. Phys.Rev., D68:105011, 2003.

[38] Dmitri Diakonov. Instantons at work. Prog.Part.Nucl.Phys., 51:173-222, 2003.

[39] Thomas Schweigler. Topological objects and chiral symmetry breaking in QCD. Master's thesis, Vienna University of Technology, 2012. 